\title{
Protective effects of heme oxygenase-1-transduced bone marrow-derived mesenchymal stem cells on reduced-size liver transplantation: Role of autophagy regulated by the ERK/mTOR signaling pathway
}

\author{
RAORAO WANG ${ }^{1 *}, Z^{*}$ ONGYANG SHEN ${ }^{2,3^{*}}$, LIU YANG $^{1}$, MINGLI YIN $^{1}$, \\ WEIPING ZHENG ${ }^{2}$, BIN WU ${ }^{2}$, TAO LIU ${ }^{2,4}$ and HONGLI SONG ${ }^{2,5}$ \\ ${ }^{1}$ Tianjin First Central Hospital Clinic Institute, Tianjin Medical University, Tianjin 300070; \\ ${ }^{2}$ Department of Organ Transplantation, Tianjin First Central Hospital; ${ }^{3}$ Tianjin Clinical Research Center \\ for Organ Transplantation; ${ }^{4}$ Key Laboratory of Emergency and Care Medicine of Ministry of Health; \\ ${ }^{5}$ Tianjin Key Laboratory of Organ Transplantation, Tianjin 300192, P.R. China
}

Received March 10, 2017; Accepted August 21, 2017

DOI: $10.3892 / \mathrm{ijmm} .2017 .3121$

\begin{abstract}
Autophagy is a critical lysosomal pathway that degrades cytoplasmic components to maintain cell homeostasis and provide substrates for energy metabolism. A study revealed that heme oxygenase-1 (HO-1)-transduced bone marrow-derived mesenchymal stem cells (BM-MSCs) could protect $50 \%$ reduced-size liver transplantation (RSLT) in a rat model. However, the mechanisms remain mostly unknown. The aim of the present study was to explore the effects and related mechanism of autophagy on the protection conferred by HO-1-transduced BM-MSCs (HO-1/BM-MSCs) on 50\% RSLT in a rat model. The authors established an acute rejection model following 50\% RSLT in rats, with recipients divided into three groups receiving treatment with BM-MSCs, HO-1/BM-MSCs or normal saline (NS) injected through the dorsal penile vein.
\end{abstract}

Correspondence to: Professor Hongli Song, Department of Organ Transplantation, Tianjin First Central Hospital, 24 Fukang Road, Nankai, Tianjin 300192, P.R. China

E-mail: hlsong26@163.com

*Contributed equally

Abbreviations: RSLT, reduced-size liver transplantation; BM-MSCs, bone marrow-derived mesenchymal stem cells; HO-1, heme oxygenase-1; HO-1/BM-MSCs, HO-1-transduced BM-MSCs; ROS, reactive oxygen species; GFP, green fluorescence protein; POD, post-operative day; LC3-I, soluble autophagy microtubuleassociated protein 1 light chain 3; PV, portal vein; IHVC, infrahepatic vena cava; SHVC, suprahepatic vena cava; mTOR, mammalian target of rapamycin

Key words: autophagy, bone marrow mesenchymal stem cells, heme oxygenase-1, reduced-size liver transplantation, ERK/mTOR signaling pathway
Transplanted liver tissues at $0,1,3,5,7,10$ and 14 days following transplantation were acquired for further analysis. The results indicated that the expression of autophagy-related proteins LC3 and Beclin-1 increased, the levels of ERK and p-ERK increased, and the levels of mammalian target of rapamycin (mTOR) and p-mTOR decreased in the HO-1/BM-MSCs. These observations indicated that autophagy is involved in the protective effects of HO-1/BM-MSCs on liver grafts following RSLT, possibly via upregulation of autophagy-related proteins through the ERK/mTOR signaling pathway.

\section{Introduction}

Liver transplantation has become the most effective treatment for end-stage liver diseases. However, a shortage of donor organs remains the major impediment to the further development of liver transplantation. Reduced-size liver transplantation (RSLT), either living-related liver transplantation or split-liver transplantation, can meet the shortage of livers to some extent (1) and has been applied widely in clinics. However, ischemia-reperfusion injury weakens the regeneration of the remnant liver after RSLT $(2,3)$. In addition, the risk of primary graft dysfunction caused by microvascular dysfunction and immune-mediated allograft rejection also increases following RSLT (4). Therefore, it is necessary to find treatments that inhibit the death and stimulate the regeneration of hepatocytes, alleviate the rejection of the transplanted liver and induce immune tolerance.

Bone marrow-derived mesenchymal stem cells (BM-MSCs) are pluripotent, can differentiate into endothelial cells for endothelial repair $(5,6)$, secrete a variety of cytokines, chemokines and growth factors promoting cell proliferation and differentiation and can migrate to the damaged tissue to repair it (7). In addition, because of their low cell surface expression of major histocompatibility complex molecules, BM-MSCs show immunomodulatory activity, which plays an important role in allograft rejection, and can induce immune tolerance 
and graft regeneration $(8,9)$. However, following transfusion, BM-MSCs present at a low concentration and short duration of activity in the target tissues, and thus, are not widely applied. Heme oxygenase-1 (HO-1) is the rate-limiting enzyme of heme catalyzation, whose activity can be induced by oxidative stress. HO-1 has antioxidant and homeostasis maintenance effects (10). HO-1 can increase the activity and prolong the duration of BM-MSCs (11). Previous studies of the authors have demonstrated that HO-1-transduced BM-MSCs (HO-1/BM-MSCs) may protect the transplanted liver by participating in the regulation of transplantation immunity and repair of the damaged liver tissue $(12,13)$. However, the mechanism of this protective effect remains unknown.

Autophagy is an important lysosomal pathway-dependent biological process in eukaryotes, which degrades cytoplasmic components to maintain cell homeostasis and provide substrates for energy metabolism (14). Autophagy plays an important role in a variety of liver functions. Firstly, as the liver has a unique regenerative ability, autophagy can clear the damaged cell organelles, and oxidized or accumulated proteins, during liver regeneration. Second, autophagy participates in and regulates metabolic pathways of proteins, carbohydrates and lipids in the liver. Furthermore, autophagy may have metabolic or proliferative effects during rapid regeneration of hepatocytes, although this requires further study (14). Studies have demonstrated that, as a cytoprotective mechanism, autophagy could inhibit oxidative stress, reduce the amount of reactive oxygen species (ROS) generated by Kupffer cells and inhibit the death of hepatocytes (14-17). Autophagy also participates in the positive and negative selection of $\mathrm{CD}^{+}{ }^{+} \mathrm{T}$ cells, and plays an important role in the central and peripheral immune tolerance to self-antigens (18). Is autophagy involved in the protection of HO-1/BM-MSCs on RSLT? The aim of the present study was to determine the participation of autophagy in the protective effects of HO-1/BM-MSCs on RSLT and to explore its possible mechanism.

\section{Materials and methods}

Animals and ethics. Specific-pathogen-free healthy adult inbred male Brown-Norway $(\mathrm{BN})$ rats $(\mathrm{n}=45)$ and male Lewis rats $(n=35)$ were purchased from the Vital River Laboratories Animal Technology, Co., Ltd. (Beijing, China). The rats were housed individually in standard animal facilities at $18-26^{\circ} \mathrm{C}$ with a 12-h light/dark cycle and were provided with commercially available chow and tap water ad libitum. BM-MSCs were extracted from syngeneic inbred male BN rats $(80-100 \mathrm{~g}$; 4-5 weeks old). Inbred male Lewis rats (210-250 g; 8-10 weeks old) were the liver transplantation donors, and inbred male BN rats (210-250 g; 8-10 weeks old) were the recipients. The difference in body weight between the donor and the recipient of each pair did not exceed $10 \mathrm{~g}$. Food was withheld from the recipient animals for $12 \mathrm{~h}$ before surgery, but was not withheld from the donor animals. The study protocol was approved by the Animal Care and Research Committee of Tianjin First Central Hospital (Tianjin, China). All surgeries and sacrifices were performed under $5 \%$ chloral hydrate anesthesia $(0.5 \mathrm{ml} / 100 \mathrm{~g})$. Every effort was made to minimize animal suffering.

Isolation, characterization and differentiation induction of BM-MSCs. BM-MSCs were isolated aseptically from the femur and tibia of 10 syngeneic male $\mathrm{BN}$ rats following sacrifice by cervical dislocation. After cutting off both ends of the epiphyseal, the marrow cavity was rinsed by DMEM/F12 (Gibco, Thermo Fisher Scientific, Inc., Waltham, MA, USA) containing $10 \%$ fetal bovine serum (FBS). Red blood cells were lysed using $0.1 \mathrm{~mol} / 1 \mathrm{NH}_{4} \mathrm{Cl}$, and the remaining cells were washed, resuspended, and cultured $1 \times 10^{6} / \mathrm{T} 75$ culture flask at $37^{\circ} \mathrm{C}$ with $5 \% \mathrm{CO}_{2}$ in Dulbecco's modified Eagle's medium (DMEM)/F12 containing $100 \mathrm{U} / \mathrm{ml}$ penicillin, $100 \mathrm{mg} / \mathrm{ml}$ streptomycin and 15\% FBS (13). The well-grown third-passage BM-MSCs were resuspended at $1 \times 10^{6} / \mathrm{ml}$ and then labeled with antibodies against CD29 (1:80, PE, 102207), CD90 (1:200, FITC, 202503), CD45 (1:80, PE, 202207), RT1A (1:80, PE, 205208), RT1B (1:200, FITC, 205305) (both from BioLegend, Inc., San Diego, CA, USA) and CD34 (1:5, FITC, sc-7324; Santa Cruz Biotechnology, Inc., Dallas, TX, USA) for 30 min for flow cytometric analysis (BD FACSAria III; BD Biosciences, Franklin Lakes, NJ, USA) of the expression of cell surface markers.

Adipogenic differentiation medium was prepared as DMEM/F12 containing 10\% FBS, $200 \mathrm{mM}$ indomethacin (Sigma-Aldrich, Merck KGaA, Darmstadt, Germany), $0.5 \mathrm{mM}$ 1-methyl-3-isobutylxanthine (Sigma-Aldrich, Merck KGaA), $40 \mathrm{U} / \mathrm{ml}$ insulin (Sigma-Aldrich, Merck KGaA) and $1 \mathrm{mM}$ dexamethasone (Sigma-Aldrich, Merck KGaA). Following staining with Oil Red O (Beijing Dingguo Changsheng Biotechnology Co., Ltd., Beijing, China), BM-MSCs were observed under a light microscope to identify red lipid droplets in the cytoplasm. Osteogenic differentiation medium was prepared as DMEM/F12 containing 10\% FBS, 1 mM dexamethasone, $1 \mathrm{M}$ sodium glycerophosphate (Sigma-Aldrich, Merck KGaA) and $100 \mathrm{ng} / \mathrm{ml}$ vitamin C (Sigma-Aldrich, Merck KGaA), with a $\mathrm{pH}$ value adjusted to 7.3-7.4. The medium was changed every $72 \mathrm{~h}$. Following staining with the von Kossa cell staining kit (Shanghai Genmed Gene Pharmaceutical Technology Co., Ltd., Shanghai, China), BM-MSCs were observed under a light microscope to identify black calcium salt in the cytoplasm.

Transduction of BM-MSCs with HO-1-bearing recombinant adenovirus. When BM-MSCs were completely adherent, HO-1-bearing recombinant adenovirus (Shanghai GeneChem Co., Ltd., Shanghai, China) at a multiplicity of infection of 10 were added into the flask to transduce Adv/HO-1/BM-MSCs; the reaction was carried out in the dark. Adenoviruses expressing the green fluorescence protein (GFP) were also added into the flask to transduce Adv/GFP/BM-MSCs to verify the expression of the target gene. After culturing for 6-8 $\mathrm{h}$, the supernatant was discarded and replaced with complete culture medium DMEM/F12 containing 10\% FBS for continued cultivation. The cells were observed under a fluorescence microscope (Olympus IX71; Olympus Corp., Tokyo, Japan) to evaluate the expression of GFP fluorescence and the morphology of nucleus at room temperature, and then were photographed using Image-Pro Plus 6.0 (Media Cybernetics, Inc., Rockville, MD, USA).

Establishment of a rejection model in 50\% RSLT of rats and the experimental protocol. A 50\% RSLT rejection model was established with Lewis donor rats and BN recipient rats, as described by Zhao et al (19). The donor livers were perfused 
via the portal vein (PV) with $4^{\circ} \mathrm{C}$ lactated Ringer's solution containing heparin sodium $(50 \mathrm{U} / \mathrm{ml})$. The harvested graft was preserved in a bath of lactated Ringer's solution at $4^{\circ} \mathrm{C}$. The cuff was slipped over the PV using microforceps, and the distal end of the vein was everted over the cuff and secured with a circumferential 5-0 silk ligature. The same procedure was performed for the infrahepatic vena cava (IHVC) cuff preparation. After the recipient liver was removed, the donor liver was placed orthotopically in the abdominal cavity of the recipient. The suprahepatic vena cava (SHVC) was anastomosed end-toend using a continuous 7-0 nylon suture. A cuff anastomosis of the PV and IHVC was then performed. The graft was reperfused by opening the PV, IHVC and SHVC in turn. The bile duct was connected by telescoping a tube in the bile duct of the donor into that of the recipient. Experimental animals were divided into three groups, which received normal saline (NS) (group 1), BM-MSCs (group 2) or HO-1/BM-MSCs (group 3). $\mathrm{HO}-1 / \mathrm{BM}-\mathrm{MSC}$ or BM-MSCs were injected through the superficial dorsal veins immediately following the surgery. Rats of the cell-treated group received $5 \times 10^{6} / \mathrm{ml}(1 \mathrm{ml})$, while the control groups were given the equivalent volume of NS. Five animals per time-point were euthanized on post-operative day (POD) $0,1,3,5,7,10$ or 14 for further analysis of the transplanted liver.

Histopathological analysis. After fixation in $10 \%$ formalin at $37^{\circ} \mathrm{C}$ for at least $48 \mathrm{~h}$, recipients' hepatic tissues were embedded in paraffin, cut into $5 \mu \mathrm{m}$ thick sections, and stained with hematoxylin and eosin (H\&E). Pathological changes and the extent of rejection were evaluated under a light microscope. Acute cellular rejection was classified according to the Banff criteria (20).

Apoptosis detection. Paraffin sections of liver tissue were routinely dewaxed, dehydrated and digested by proteinase $\mathrm{K}$ (Promega Corp., Madison, WI, USA). Terminal deoxynucleotidyl transferase dUTP nick end labeling (TUNEL) reaction mixture (Promega) was added dropwise and incubated at $37^{\circ} \mathrm{C}$ for $1 \mathrm{~h}$. The average number of apoptotic cells was analyzed in 10 randomly selected visual fields using fluorescence microscopy (magnification, x200; Nikon Ni-U; Nikon Corp., Tokyo, Japan), and the mean number of apoptotic cells was measured by staining cell nuclei wih DAPI (Beijing Solarbio Science \& Technology Co., Ltd., Beijing, China) to determine the total number of cells.

Electron microscope analysis. Transplanted liver tissues were obtained from the recipient freshly, cut into $2 \times 3 \mathrm{~mm}$ samples, and fixed in $2.5 \%$ glutaraldehyde solution. Embedded sections were observed under a transmission electron microscopy (Hitachi H-600; Hitachi, Ltd., Tokyo, Japan) for the ultrastructures of the transplanted liver tissue.

Immunohistochemical analysis. Transplanted liver tissues were fixed on glass slides, heated at $70^{\circ} \mathrm{C}$ for $1 \mathrm{~h}$, deparaffinized by dimethylbenzene, hydrated by gradient ethanol, subjected to antigen retrieval, and blocked with $10 \%$ goat serum (Shanghai GeneChem; www.genechem.com.cn.html). Following incubation with primary antibodies Beclin-1 (1:500, ab55878) and LC3 A/B (1:500, \#12741), staining by diaminobenzidine and hematoxylin, and dehydration by gradient ethanol, the sections were covered with neutral balsam (Shanghai Yiyang Instrument Co., Ltd., Shanghai, China; http://yiyang17.com/index. php) according to the manufacturer's instructions, and then observed under a light microscope.

Detection of protein levels by western blotting. Radioimmunoprecipitation assay lysis buffer (Beijing Solarbio Science $\&$ Technology) was added to frozen liver tissues, which were then homogenized, and centrifuged at 12,000 x $\mathrm{g}$ for $4 \mathrm{~min}$ at $4^{\circ} \mathrm{C}$ to extract the proteins. A bicinchoninic acid assay (Wuhan Boster Biological Technology, Ltd., Wuhan, China; http://www.boster.com.cn/) was used to determine the protein content. SDS-PAGE (10\%) was used for Beclin-1, ERK and p-ERK; $8 \%$ SDS-PAGE was used for mammalian target of rapamycin (mTOR) and p-mTOR; and 15\% SDS-PAGE was used for LC3 A/B. A total of $30 \mu \mathrm{g}$ proteins each well in the gels were wet transferred to nitrocellulose membranes for $2 \mathrm{~h}$, blocked by $5 \%$ skimmed milk for $2 \mathrm{~h}$, and then incubated at $4^{\circ} \mathrm{C}$ overnight with antibodies recognizing Beclin-1 (ab55878) and GAPDH (ab8245) (both from Abcam, Cambridge, UK), ERK (\#4695) and LC3 A/B (\#12741) (both from Cell Signaling Technology, Inc., Danvers, MA, USA), p-ERK (RT1206; Hangzhou HuaAn Biotechnology Co., Ltd., Hangzhou, China), mTOR (ab32028; Abcam) and p-mTOR (sc-293132; Santa Cruz Biotechnology, Inc., Dallas, TX, USA). The antibodies were used at the following dilutions: Beclin-1 (1:500), GAPDH (1:5,000), ERK (1:1,000), p-ERK (1:250), mTOR $(1: 1,000)$, p-mTOR $(1: 100)$ and LC3 A/B $(1: 1,000)$. The membranes were then rinsed, incubated with secondary antibodies HRP-conjugated anti-rabbit IgG (1:5,000, ab191866) and HRP-conjugated anti-rat (1:2,000, ab131368) (both from Abcam) for $2 \mathrm{~h}$, rinsed again, and then visualized using the enhanced chemiluminescence system (Wuhan Boster Biological Technology). The abundance of the target protein was calculated relative to the abundance of the internal control protein, GAPDH, using a gel imaging analysis system (Alpha Innotech FluorChem FC2; Alpha Innotech Corp., San Leandro, CA, USA).

Detection of gene levels by reverse transcription-quantitative polymerase chain reaction $(R T-q P C R)$. Total RNA was extracted from the liver tissue using RNAiso Plus reagents (Takara Bio, Inc., Shiga, Japan). cDNA was reverse transcribed using reverse transcription kits (Tiangen Biotech Co., Ltd., Beijing, China) and $2 \mu \mathrm{l}$ cDNA was added to $20 \mu \mathrm{l}$ of a PCR reaction system using a fluorescence quantitative PCR kit (Sangon Biotech Co., Ltd., Shanghai, China; http://www. sangon.com/) for amplification. Primers were synthesized by Sangon Biotech. The primer sequences were as follows: ERK sense, 5'-GGCAACCGCCATTTCTCG-3' and antisense, 5'-GCTTCGCTTCTGTTTAGCATCAC-3'; mTOR sense, 5'-TTTGGACGGTGTAGAACTTGGAG-3' and antisense, 5'-CGAACCCTGTTAATAATCTGAATAGC-3'; and GAPDH sense, 5'-CGTATCGGACGCCTGGTTAC-3' and antisense, 5'-GGATCTCGCTCCTGGAAGATG-3'. The reaction conditions were $95^{\circ} \mathrm{C}$ pre-denaturation for $30 \mathrm{sec}$, and 40 cycles of PCR amplification comprising $95^{\circ} \mathrm{C}$ for $10 \mathrm{sec}, 58^{\circ} \mathrm{C}$ for $30 \mathrm{sec}$ and $72^{\circ} \mathrm{C}$ for $30 \mathrm{sec}$. The results were analyzed by a LightCycler ${ }^{\circledR} 96$ real-time fluorescence quantitative PCR 

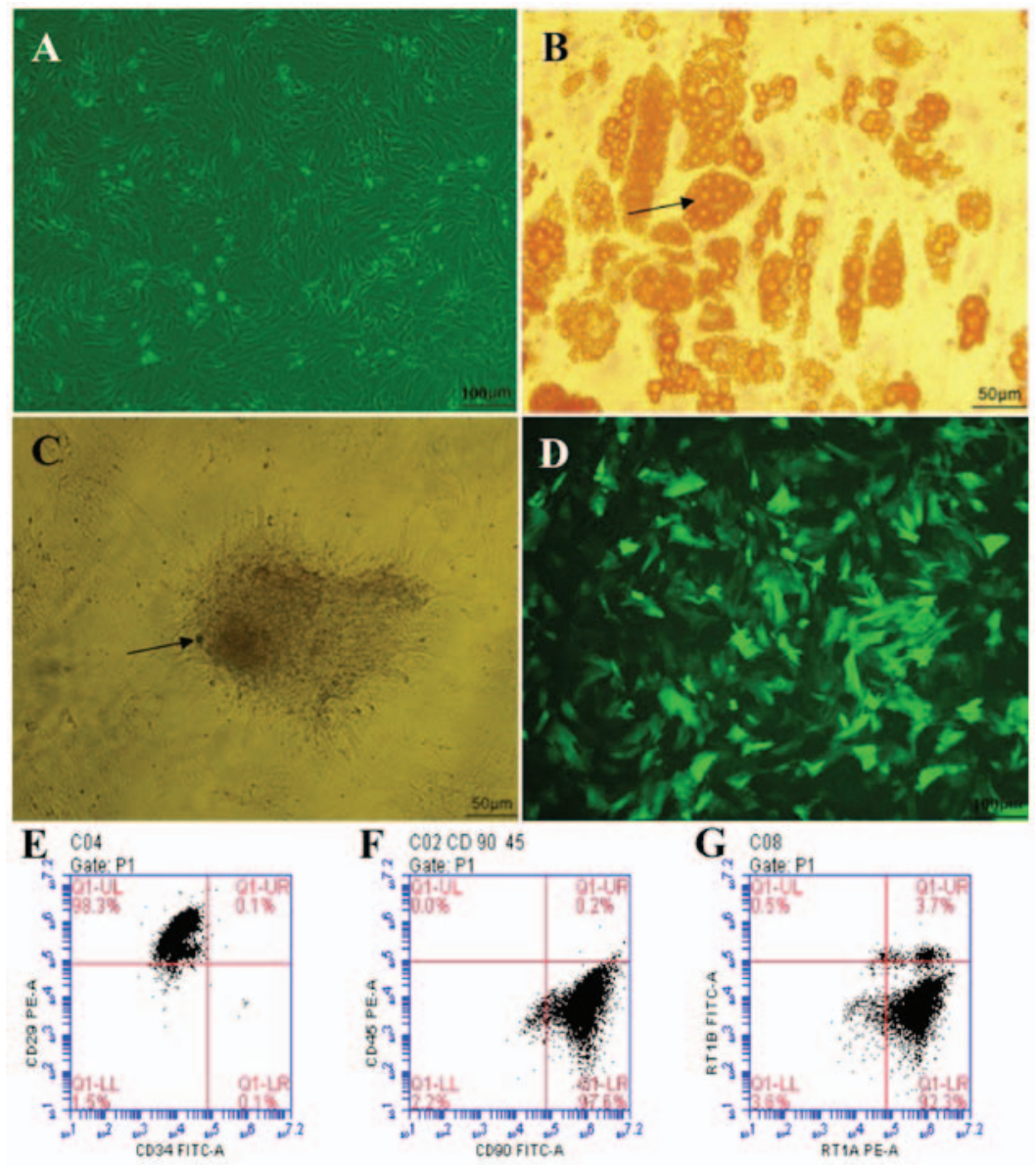

Figure 1. Morphological characteristics, differentiation induction and phenotypic identification of BM-MSCs. (A) Bright field image of third-passage BM-MSCs following transduction with HO-1, growing as long spindle-shapes with a whirlpool or paralleled pattern (magnification, x100). (B) Adipogenic induction (magnification, $\mathrm{x} 200$ ), the arrow presents the orange lipid droplets in the cytoplasm after staining with Oil Red O. (C) Osteogenic induction (magnification, x200), the arrow indicates the black calcium salt deposit after von Kossa staining. (D) Fluorescent image of third-passage BM-MSCs following transduction with HO-1, showing that $\sim 85 \%$ of the cells are transduced with HO-1 (emitting green fluorescence; magnification, x100). (E) $98.3 \%$ of the cells

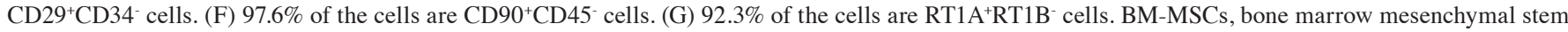
cells; HO-1; heme oxygenase-1.

detection system (Roche Diagnostics GmbH, Basel, Switzerland) (21).

Statistical analysis. The SPSS software (version, 17.0; SPSS, Inc., Chicago, IL, USA) was used for statistical analysis. The GraphPad Prism 5.0 software (GraphPad Software, Inc., La Jolla, CA, USA) was used to plot data for presentation. Normally distributed data were presented as means \pm standard deviation. Different groups of data were compared by the t-test or analysis of variance (ANOVA). $\mathrm{P}<0.05$ was considered to indicate a statistically significant difference.

\section{Results}

Morphology, differentiation induction and phenotypic analysis of BM-MSCs. Isolated primary BM-MSCs gradually became adherent after $12 \mathrm{~h}$, and impurities such as hematopoietic cells became fewer and fewer with each medium exchange and passage. The third-passage cells were obtained after $\sim 15$ days, and grew as long spindle-shaped whirlpool or paralleled pattern cells, which are the typical morphological characteristics of BM-MSCs (Fig. 1A). Meanwhile, isolated BM-MSCs could be induced to differentiate into adipocytes and osteoblasts in adipogenic and osteogenic differentiation medium, respectively (Fig. 1B and C). Phenotypic examination of the third-passage BM-MSCs surface markers by flow cytometry demonstrated that $>92 \%$ of these cells were positive for CD29, CD90 and RT1A, and negative for CD34, CD45 and RT1B (Fig. 1E-G). These results suggested that, following three passages of culture, the BM-MSCs were pure and had typical characteristics.

Transduction of BM-MSCs with HO-1-bearing recombinant adenovirus. BM-MSCs were transduced with HO-1-bearing recombinant adenoviruses carrying GFP, at a multiplicity of infection of 10 . After $48 \mathrm{~h}$, the GFP fluorescence emitted by Adv-BM-MSCs was observed under a fluorescence microscope, and the proportion of adenovirus-infected BM-MSCs emitting green fluorescent was $\sim 85 \%$ (Fig. 1D), which suggested that most BM-MSCs were successfully transduced with HO-1.

Histopathological characteristics and rejection activity index of transplanted liver after RSLT. The recipients treated with NS reported minor damage immediately after RSLT, which then progressed with little inflammation periportally 

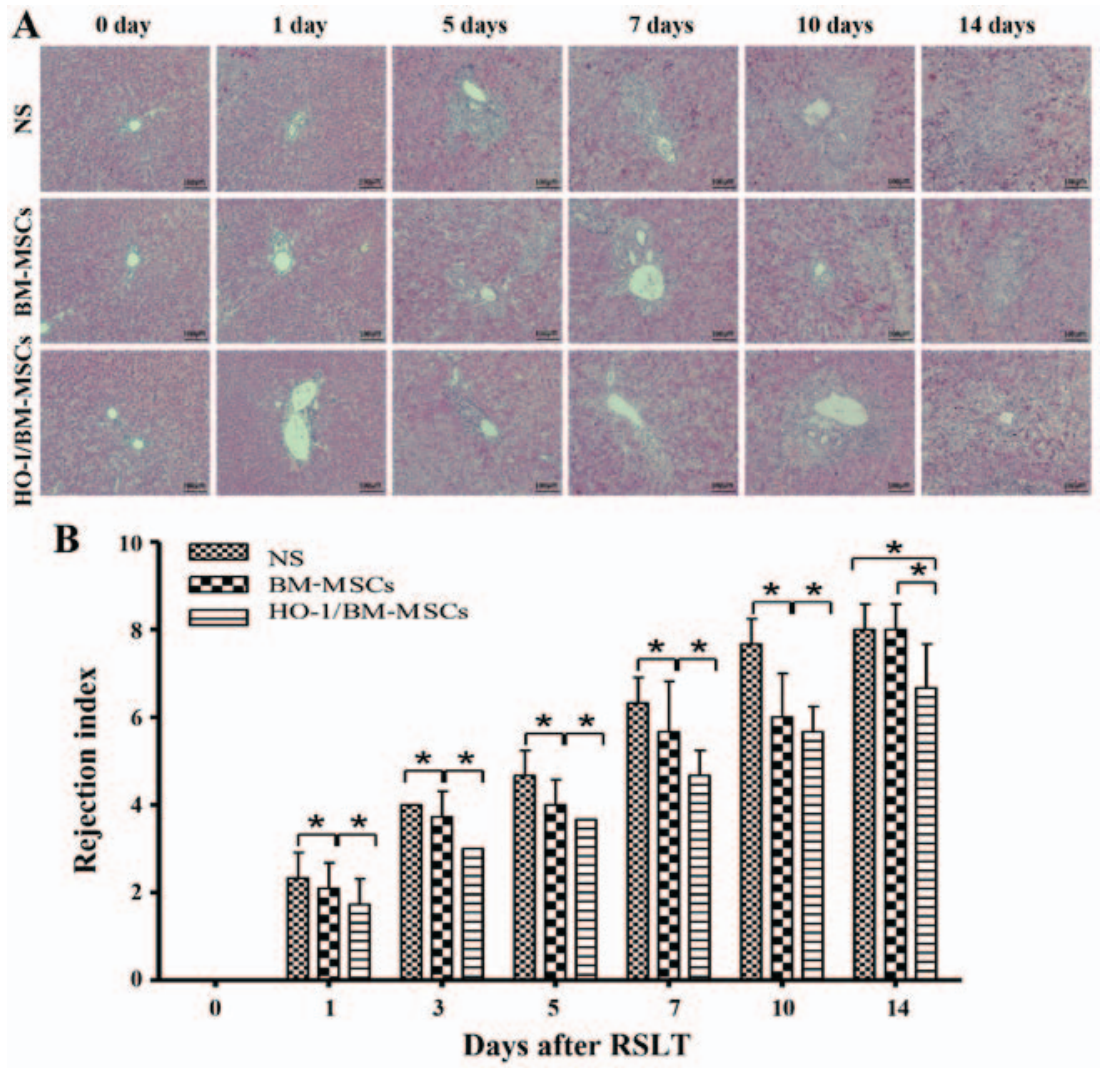

Figure 2. Histopathological characteristics and rejection activity index of transplanted livers after RSLT (magnification, $\mathrm{x} 100$ ). (A) The acute rejection in the normal saline (NS)-treated group became progressively aggravated post transplantation. Periportal lymphocytes infiltration increased gradually to significant levels, interlobular bile ducts showed inflammation and subendothelial lymphocytic infiltration involving the interlobular vein and central vein progressed significantly. The acute rejection was moderate to severe on POD 7, POD 10 and POD 14. Portal areas were infiltrated with a large number of mixed lymphocytes, which extended significantly to the surrounding parenchyma, interlobular bile ducts were infiltrated with inflammatory cells with severe biliary epithelial injury and subendothelial lymphocytic infiltration involving the interlobular vein and central vein was significant. The acute rejection in the BM-MSCs-treated group also became progressively aggravated post transplantation, however, it was less severe than that of the NS-treated group at each time-point, except on POD 14. Little lymphocyte infiltration was visible periportally on POD 7. The portal areas were infiltrated with a large number of lymphocytes, with aggravated biliary inflammation and periphlebitis on POD 14. The acute rejection in the HO-1/BM-MSCs-treated group also became increasingly aggravated post transplantation; however, it was less severe than that of the NS-treated group and the BM-MSCs-treated group. The rejection was mild at POD 7, with little lymphocyte infiltration periportally, scarcely any biliary epithelium degeneration and cholangitis. The acute rejection in the HO-1/BM-MSCs-treated group deteriorated after POD 7, but was still less severe than that of the NS-treated group and the BM-MSCs-treated group. (B) RAI in the different groups. The RAI of the BM-MSCs-treated group was significantly lower than that of the NS-treated group at each time-point except on POD 14 and the RAI of the HO-1/BM-MSCs-treated group was significantly lower than that of the NS-treated group and the BM-MSCs-treated group at each time-point. n=5 at each time-point for each group. "P<0.05 as indicated. RSLT, reduced-size liver transplantation; POD, post-operative day; BM-MSCs, bone marrow mesenchymal stem cells; HO-1/BM-MSCs, HO-1 transduced BM-MSCs; RAI, rejection activity index.

(mainly lymphocyte infiltration) without significant expansion of the portal tracts, and lymphocyte infiltration beneath venous endothelium was not obvious on POD 1. Periportal inflammation was obvious on POD 3, primarily because of lymphocyte infiltration, and lymphocyte infiltration beneath the venous endothelium was still not evident. The acute rejection increased on POD 5, with mixed inflammatory infiltration (including lymphoblasts and eosinophils) of the portal tracts spreading to the surrounding parenchyma; most interlobular bile ducts were infiltrated by inflammatory cells, and subendothelial lymphocytic infiltration involved the interlobular vein. Acute rejection increased sharply on POD 7, with moderate to severe acute rejection being observed on POD 7, POD 10 and POD 14. Most of the portal areas were infiltrated with a large number of mixed lymphocytes, which extended to the surrounding parenchyma significantly. Interlobular bile ducts presented inflammation and luminal disruption; subendothelial lymphocytic infiltration involving the interlobular vein and central vein was significant and perivenular hepatocyte necrosis was observed. The rejection was mild in BM-MSCstreated group on POD 0, POD 1, POD 3 and POD 5 compared with that of the NS-treated group, with little visible lymphocyte infiltration periportally, and cholangitis and degeneration of the biliary epithelium was scarcely seen; interlobular and central vein phlebitis was uncommon. However, acute rejection in the BM-MSCs-treated group increased sharply on POD 7, but was less severe than that of the NS-treated group. The liver deteriorated to serious injury on POD 14, with no significant difference compared with the NS-treated group. The rejection was mild in HO-1/BM-MSCs-treated group on POD 0, POD 1, POD 3 and POD 5 compared with that of the NS-treated group and the BM-MSCs-treated group. The condition of the livers deteriorated gradually after POD 7, but was less severe than that of the NS-treated group and the BM-MSCs-treated group on POD 7, POD 10 and POD 14. These results suggested that the rejection model after RSLT was constructed successfully, and that the acute rejection in the HO-1/BM-MSCs-treated group was less severe compared 

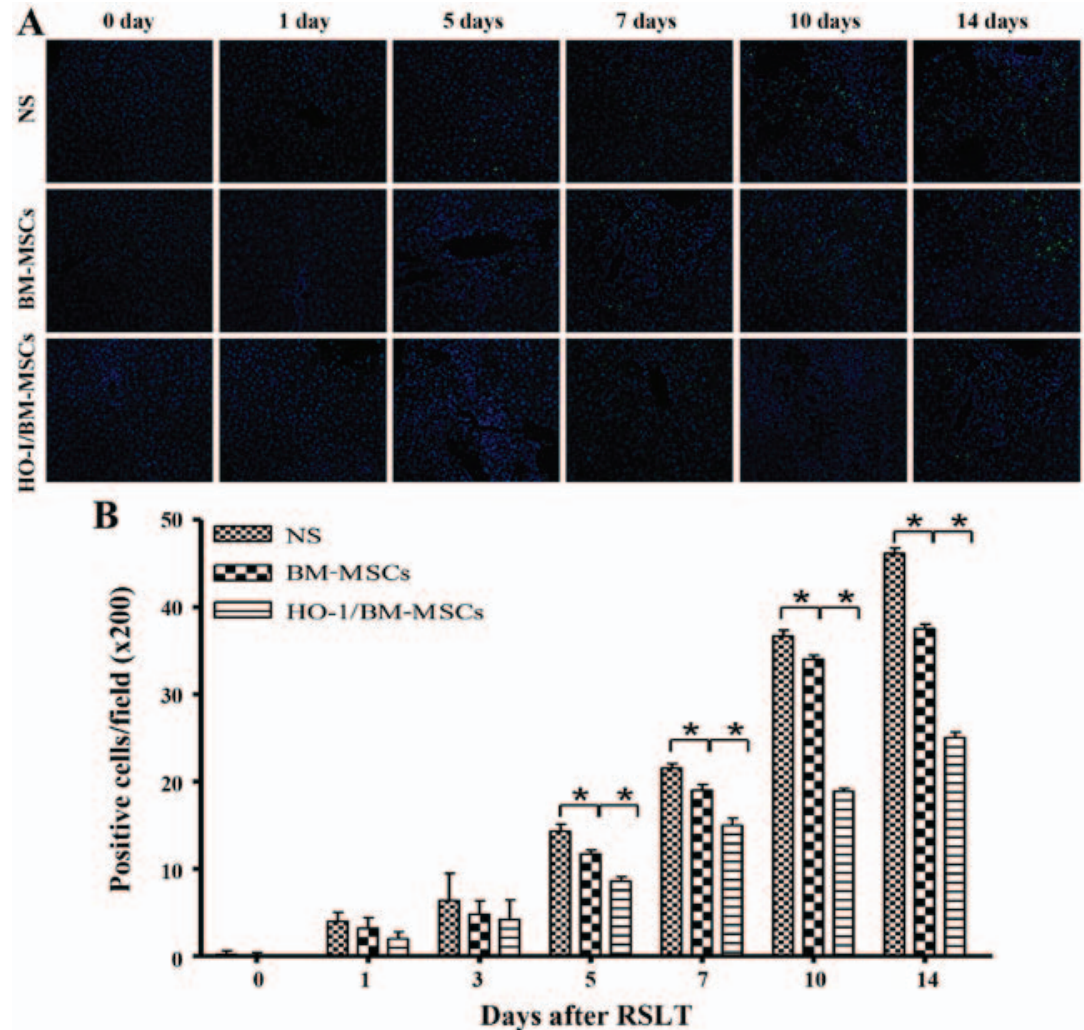

Figure 3. Apoptosis in the transplanted liver tissues (magnification, x200). (A and B) Terminal deoxynucleotidyl transferase dUTP nick end labeling staining positive cells appear green and represent apoptotic cells, and DAPI-labeled nuclei appear blue. Apoptotic cells were scattered in the transplanted liver on POD 0 and POD 1, with no significant differences between the three groups. The numbers of apoptotic cells in the HO-1/BM-MSCs-treated group were significantly lower than those in the normal saline (NS)-treated group on POD 5, POD 7, POD 10 and POD 14. "P<0.05 as indicated. POD, post-operative day; HO-1/BM-MSCs, HO-1 transduced BM-MSCs.

NS

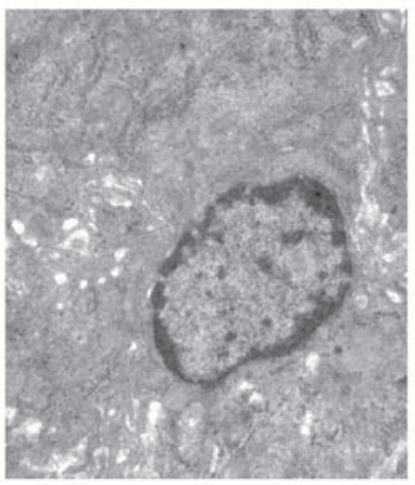

BM-MSCs

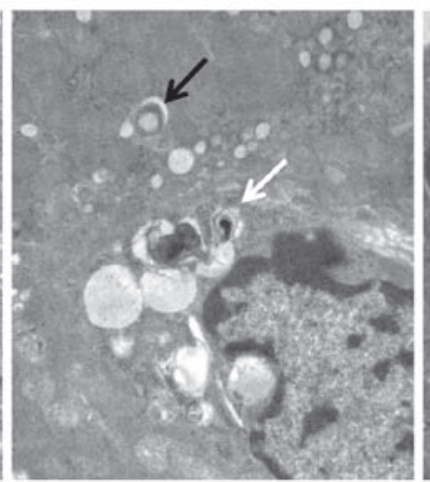

HO-1/BM-MSCs

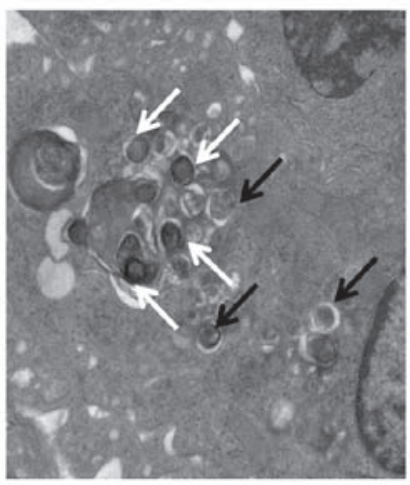

Figure 4. Ultrastructure and autophagic vacuoles of the transplanted liver on POD 7 observed under a transmission electron microscope (magnification, $\mathrm{x} 25,000)$. The normal saline (NS)-treated group presented nuclear pycnosis, an irregular nuclear membrane with deformation, significantly swollen endoplasmic reticulum and mitochondria and no typical autophagic vacuoles. The BM-MSCs-treated group showed slight nuclear pycnosis and deformation, no obvious edema of the endoplasmic reticulum, slight edema of the mitochondria, and visible initial and degraded autophagic vacuoles. The HO-1/BM-MSCstreated group reported slight nuclear pycnosis without deformation, no edema in endoplasmic reticulum or mitochondria, and a large number of initial autophagic vesicles and degraded autophagic vesicles. The black arrow shows initial autophagic vesicles, containing complete rough endoplasmic reticulum and mitochondria. The white arrow shows degraded autophagic vesicles, containing degraded rough endoplasmic reticulum and mitochondria. POD, postoperative day; BM-MSCs, bone marrow mesenchymal stem cells; HO-1/BM-MSCs, HO-1 transduced BM-MSCs.

with that of the BM-MSCs-treated group and the NS-treated group at different time-points after RSLT and the duration of action was long-lasting (Fig. 2A).

When the degree of rejection was classified by the rejection activity index (RAI) using the Banff scheme, the RAIs of the HO-1/BM-MSCs-treated group were significantly lower than those of the NS and BM-MSCs-treated groups at each timepoint $(\mathrm{P}<0.05)$. The RAIs of the BM-MSCs-treated group were significantly lower than those of the NS-treated group at each time-point, except on POD $14(\mathrm{P}<0.05)$. These results suggested that HO-1/BM-MSCs treatment could reduce acute rejection injury after RSLT, and the duration of action could 

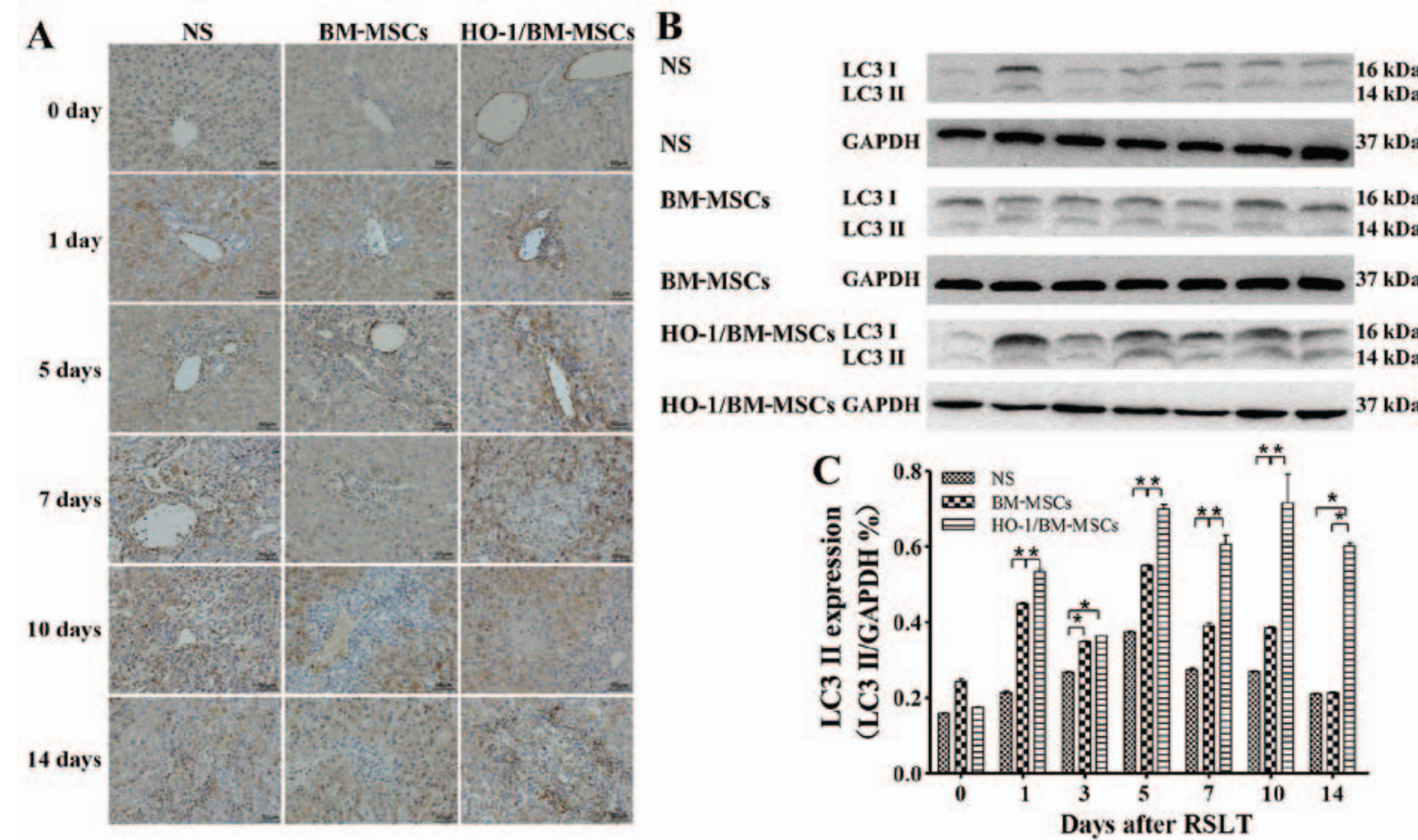

Figure 5. Expression of autophagy-related protein LC3 I/II following RSLT. (A) Expression of LC3 I/II protein immunohistochemically on POD 0, POD 1 , POD 5, POD 7, POD 10 and POD 14. Only a small amount of LC3 I/II protein was present in endothelia of the central vein on POD 0, which was similar in all three groups. The LC3 I/II protein was also visible in cytoplasm of hepatocytes on POD 1. The protein abundance increased significantly after POD 5 , appearing in the cytoplasm of most central venous endothelia and hepatocytes. The overall expression in the HO-1/BM-MSCs-treated group was the highest and was much higher than that in the normal saline (NS)-treated group and in the BM-MSCs-treated group. (B and C) Expression of the LC3 II protein as demonstrated by western blotting on POD 0, POD 1, POD 3, POD 5, POD 7, POD 10 and POD 14. The protein level in the HO-1/BM-MSCs-treated group was significantly higher than that of the NS-treated group and BM-MSCs-treated group at each time-point, except on POD 0 and POD 3 . "P<0.05 as indicated. RSLT, reduced-size liver transplantation; POD, post-operative day; HO-1/BM-MSCs, HO-1 transduced BM-MSCs; BM-MSCs, bone marrow mesenchymal stem cells.

extend to POD 14, which was longer than that achieved by simple BM-MSCs treatment (Fig. 2B).

Apoptosis in transplanted liver tissue. Apoptotic cells were scattered in all of the three groups of transplanted livers on POD 0 and 1. The numbers of apoptotic cells increased progressively on POD 5, POD 7, POD 10 and POD 14, but were significantly lower in the HO-1/BM-MSCs-treated group than in the NS and BM-MSCs-treated groups. These results indicated that HO-1/BM-MSCs could reduce the apoptosis of transplanted liver tissue (Fig. 3).

Ultrastructure and autophagic vacuoles of the transplanted liver on POD 7. The autophagic vacuoles were surrounded by a bilayer membrane, and the bilayer was parallel, with a narrow gap. The initial autophagic vacuoles contained complete mitochondria or endoplasmic reticulum, and the degraded autophagic vacuoles contained degraded rough endoplasmic reticulum or mitochondria, which could be recognized by the different inclusions. In the NS-treated group on POD 7, the nucleus was condensed significantly, and the endoplasmic reticulum and mitochondria showed obvious edema, without significant autophagic vacuoles. In the BM-MSCs-treated group, the nucleus was condensed slightly, with visible autophagic vacuoles. By contrast, in the HO-1/BM-MSCs-treated group, there was no nuclear condensation or distortion, and a large number of autophagic vacuoles were observed. These results suggested that the ultrastructural damage of HO-1/BM-MSCs-treated livers on POD 7 was milder than that of the NS and BM-MSCs-treated groups, and the number of initial and degraded autophagic vacuoles in the HO-1/BM-MSCs-treated group was higher than that in the NS and BM-MSCs-treated groups (Fig. 4).

\section{Levels of autophagy-related proteins LC3 I/II and Beclin-I after RSLT}

Levels of the LC3 I/II protein. Immunohistochemical tests presented only a small amount of LC3 I/II protein in the endothelia of the central vein in all three groups on POD 0 , with no significant difference between the three groups. The LC3 I/II protein was visible in the cytoplasm of both the central venous endothelia and hepatocytes on POD 1, with the highest level of the protein in the HO-1/BM-MSCs-treated group. The amount of LC3 I/II protein increased after POD 5 as the time after RSLT was prolonged, and the overall level in the HO-1/BM-MSCs-treated group was much higher than that in the NS and the BM-MSCs-treated groups (Fig. 5A).

Western blotting indicated that the LC3 II protein level was relative low in all three groups on POD 0 , with no significant difference between them. The protein level in the HO-1/BM-MSCs-treated group on POD 1 was significantly higher than that in the NS and the BM-MSCs-treated groups $(\mathrm{P}<0.05)$. The protein level in the HO-1/BM-MSCs-treated group on POD 3 was significantly higher than that of the 

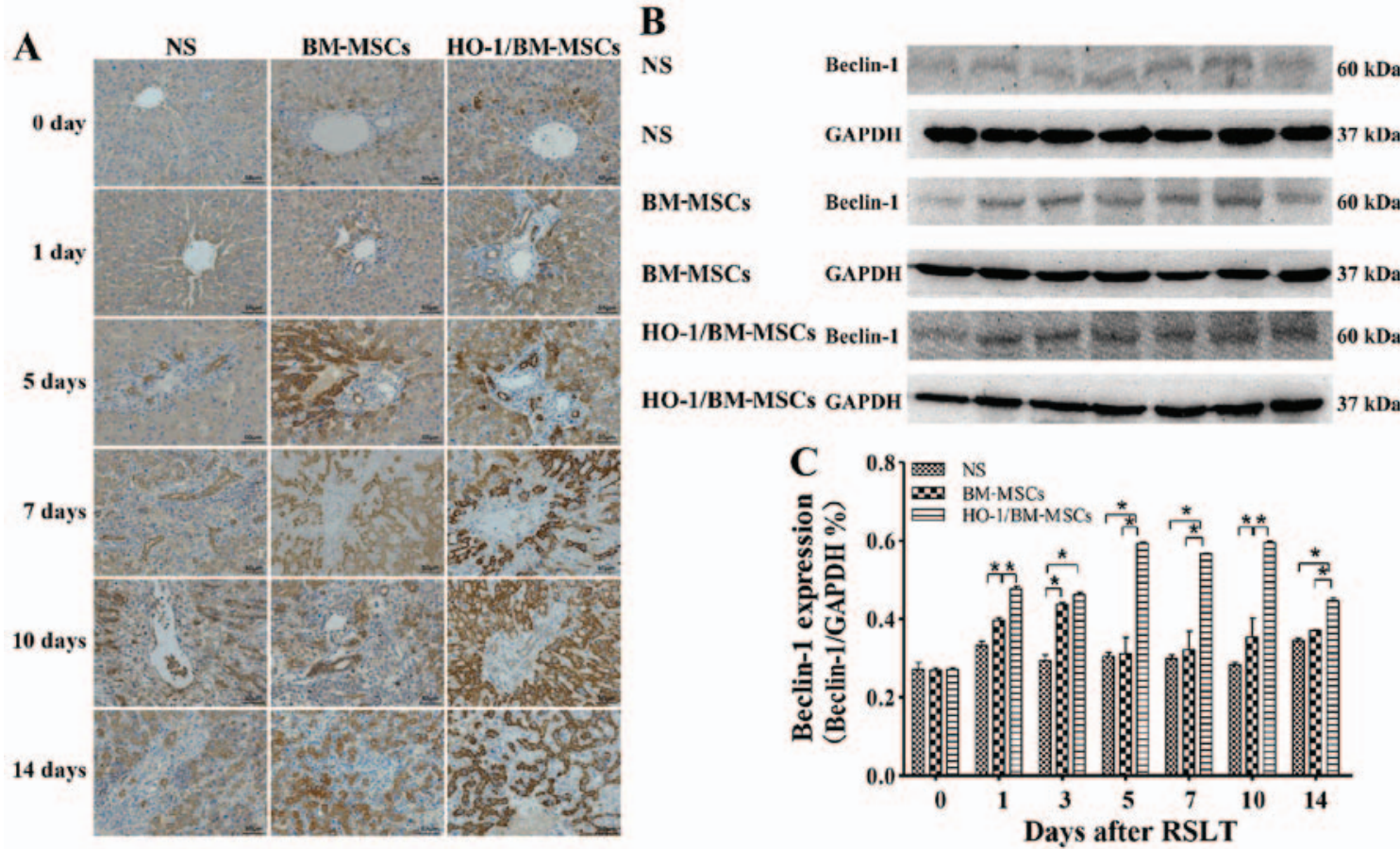

Figure 6. Levels of autophagy-related protein Beclin-1 after RSLT. (A) Levels of Beclin-1 protein as assessed immunohistochemically on POD 0, POD 1, POD 5, POD 7, POD 10 and POD 14. Only a small amount of Beclin-1 protein was present in the cytoplasm of hepatocytes around the central vein on POD 0, which was similar in all three groups. Beclin-1 could also be observed in the biliary epithelia on POD 1. The protein expression increased after POD 5, extending to the cytoplasm of most hepatocytes and biliary endothelia. The overall protein level in the HO-1/BM-MSCs-treated group was the highest and was much higher than that in the normal saline (NS)-treated group and the BM-MSCs-treated group. (B and C) Levels of the Beclin-1 protein as demonstrated by western blotting on POD 0, POD 1, POD 3, POD 5, POD 7, POD 10 and POD 14. The protein abundance in the BM-MSCs-treated group was significantly higher than that in the NS-treated group at each time-point, except on POD 5, POD 7 and POD 14. The protein abundance in the HO-1/BM-MSCs-treated group was significantly higher than that in the NS-treated group and BM-MSCs-treated group at each time-point, except on POD 0 . "P<0.05 as indicated. RSLT, reduced-size liver transplantation; POD, post-operative day; HO-1/BM-MSCs, HO-1 transduced BM-MSCs; BM-MSCs, bone marrow mesenchymal stem cells.

NS-treated group $(\mathrm{P}<0.05)$, but reported no statistically significant difference with that of the BM-MSCs-treated group. The protein level increased significantly on POD 5, POD 7, POD 10 and POD 14, especially in the HO-1/BM-MSCs-treated group. The protein level in the BM-MSCs-treated group on POD 5, POD 7 and POD 10 was significantly higher than that of the NS-treated group $(\mathrm{P}<0.05)$, and the protein level in the HO-1/BM-MSCs-treated group was significantly higher than that of the BM-MSCs-treated group $(\mathrm{P}<0.05)$ except on POD 0 and POD 3. These results demonstrated that the level of autophagy-related protein LC3 I/II in the HO-1/BM-MSCstreated group was the highest (Fig. 5B and C).

Levels of the Beclin-1 protein. Immunohistochemical tests presented only a small amount of Beclin-1 protein in the cytoplasm of hepatocytes around the central vein in all three groups on POD 0, with no significant difference between the three groups. Beclin-1 could also be observed in the biliary epithelia on POD 1. The Beclin-1 level increased after POD 5 as the time after RSLT prolonged, and the overall Beclin-1 level in the HO-1/BM-MSCs-treated group was much higher than that in the NS and the BM-MSCs-treated groups (Fig. 6A).

Western blotting demonstrated that the amount of Beclin-1 protein was relatively low in all three groups on POD 0, with no significant difference between them. The Beclin-1 level in the HO-1/BM-MSCs-treated group on POD 1 was significantly higher than that in the NS and the
BM-MSCs-treated groups $(\mathrm{P}<0.05)$. The Beclin-1 level in the HO-1/BM-MSCs-treated group on POD 3 was significantly higher than that in the NS-treated group $(\mathrm{P}<0.05)$, but reported no statistically significant difference with that of the BM-MSCs-treated group. The Beclin-1 level increased significantly on POD 5, POD 7, POD 10 and POD 14, especially in the HO-1/BM-MSCs-treated group. The Beclin-1 level in the HO-1/BM-MSCs-treated group was significantly higher than that of the NS and BM-MSCs-treated group $(\mathrm{P}<0.05)$. These results indicated that the HO-1/BM-MSCs-treated group produced the highest amount of the autophagy-related protein Beclin-1 (Fig. 6B and C).

Autophagy regulated by the ERK/mTOR signaling pathway is involved in the regulation of the protective effects of HO-1/BM-MSCs on RSLT. The amount of ERK mRNA in the HO-1/BM-MSCs-treated group was significantly higher than that of the NS-treated group at each time-point, and was significantly higher than that in the BM-MSCs-treated group, except on POD 0 and POD $10(\mathrm{P}<0.05)$. The amount of p-ERK protein in the HO-1/BM-MSCs-treated group on POD 0 was significantly higher than that in the NS-treated group $(\mathrm{P}<0.05)$, but demonstrated no significant difference with the BM-MSCs-treated group. The amount of p-ERK protein in the HO-1/BM-MSCs-treated group was significantly higher than that in the NS and BM-MSCs-treated groups on POD 1 and POD $3(\mathrm{P}<0.05)$. The overall amount of p-ERK 

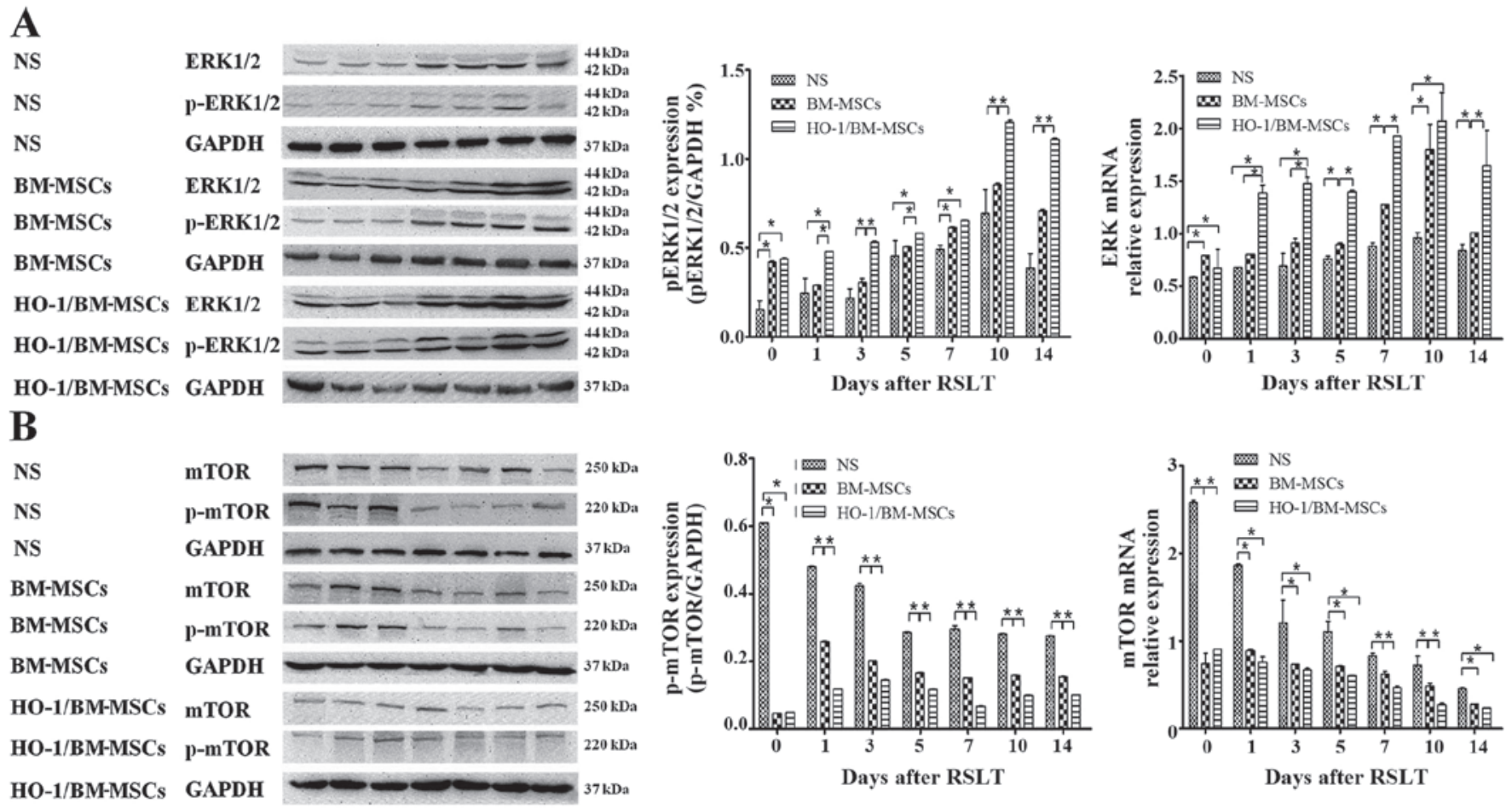

Figure 7. Expression of ERK1/2, p-ERK1/2, mTOR and p-mTOR after reduced-size liver transplantation. (A) Relative content of ERK1/2 to p-ERK1/2, as measured by RT-qPCR and western blotting. The amount of $E R K$ mRNA in the HO-1/BM-MSCs-treated group was significantly higher than that of the normal saline (NS)-treated group at each time-point, and that in the HO-1/BM-MSCs-treated group was significantly higher than that of the BM MS treatedgroup except on POD 0 and POD 10. The amount of p-ERK1/2 in the BM-MSCs-treated group was significantly higher than that in the NS-treated group on POD 0, POD 3, POD 7, POD 10 and POD 14. The amount of p-ERK1/2 in the HO-1/BM-MSCs-treated group was significantly higher than that of the BM-MSCs-treated group at each time-point, except on POD 0 and POD 7, and was significantly higher than that in the NS-treated group at each time-point. (B) Relative content of mTOR to p-mTOR, as measured by RT-qPCR and western blotting. The amount of $m T O R$ mRNA in the HO-1/BM-MSCs-treated group was significantly lower than that of the NS-treated group at each time-point, and that in the HO-1/BM-MSCs-treated group was significantly lower than the BM-MSCs-treated group except on POD 7 and POD 10. The amount of p-mTOR in the BM-MSCs-treated group was significantly lower than that in the NS-treated group at each time-point. The level of p-mTOR in the HO-1/BM-MSCs-treated group was significantly lower than that in the BM-MSCs-treated group at each time-point, except on POD 0, and was significantly lower than that in the NS-treated group at each time-point. "P $<0.05$ as indicated. ERK, extracellular signal-regulated kinase; mTOR, mammalian target of rapamycin; HO-1/BM-MSCs, HO-1 transduced BM-MSCs; POD, post-operative day; RT-qPCR, reverse transcription-quantitative polymerase chain reaction.

protein increased significantly on POD 5, POD 7, POD 10 and POD 14, especially in the HO-1/BM-MSCs-treated group. The amount of p-ERK in the HO-1/BM-MSCs-treated group was significantly higher than that of the NS-treated group at each time-point $(\mathrm{P}<0.05)$. The amount of $\mathrm{p}$-ERK in the HO-1/BM-MSCs-treated group was significantly higher than that of the BM-MSCs-treated group at each time-point, except on POD 7 (P<0.05; Fig. 7A).

The amount of $m$ TOR mRNA in the HO-1/BM-MSCstreated group was lower than that of the NS-treated group at each time-point, and that in the HO-1/BM-MSCs-treated group was lower than the BM-MSCs-treated group on POD 7 and POD 10. The amount of p-mTOR protein in the HO-1/BM-MSCs-treated group on POD 0 was significantly lower than that of the NS and BM-MSCs-treated groups $(\mathrm{P}<0.05)$. The amount of $\mathrm{p}$-mTOR protein in the HO-1/BM-MSCs-treated group was also significantly lower than that in the NS and BM-MSCs-treated groups on POD 1 and POD $3(\mathrm{P}<0.05)$. The overall amount of $\mathrm{p}-\mathrm{mTOR}$ protein decreased significantly on POD 5, POD 7, POD 10 and POD 14, especially in the HO-1/BM-MSCs-treated group. The amount of p-mTOR in the HO-1/BM-MSCs-treated group was significantly lower than that of the NS and BM-MSCs-treated groups $(\mathrm{P}<0.05)$. These results suggested that the amount of $\mathrm{p}$-ERK protein increased, while the amount of $\mathrm{p}$-mTOR protein decreased in the HO-1/BM-MSCs-treated group, which suggested that the protective effects of HO-1/BM-MSCs on RSLT may involve autophagy regulated by the ERK/mTOR signaling pathway (Fig. 7B).

\section{Discussion}

Although RSLT could alleviate the problem of organ shortage for liver transplantation to some extent (1), it also weakens the regenerative ability of the transplanted liver $(2,3)$. Previous studies have indicated that BM-MSCs could inhibit the death of hepatocytes via paracrine or direct differentiation into hepatocytes, which promotes the regeneration of the liver after injury (22-24). HO-1, also known as a 'stop signal' of inflammation, has anti-inflammatory and anti-apoptotic effects (25). $\mathrm{HO}-1$ is the inducible form of the $\mathrm{HO}$ family, and is the ratelimiting enzyme that catalyzes heme degradation to $\mathrm{CO}$, iron and biliverdin. The main function of HO-1 is to protect cells under oxidative stress and other stimuli. Many factors, such as infection, inflammation and hypoxia induce HO-1 (26). The pathological and TUNEL analysis of transplanted livers in the 
current study indicated that HO-1/BM-MSCs could promote liver regeneration and suppress rejection of the transplanted liver significantly, with better protective effects, which was consistent with our previous studies $(12,13)$; however, the exact mechanism remained unclear. The authors observed autophagic vesicles in the ultrastructure of transplanted livers after RSLT, which were more obvious in the HO-1/BM-MSCstreated group than in the BM-MSCs-treated group and NS-treated group. Therefore, to expand on the previous studies, the authors investigated whether autophagy is involved in the protective effects of $\mathrm{HO}-1 / \mathrm{BM}-\mathrm{MSC}$ on the transplanted liver following RSLT.

Soluble autophagy microtubule-associated protein 1 light chain 3 (LC3 I) is converted to LC3 II during the induction of the autophagosome, and LC3 II is involved in the formation of the autophagosome membrane. Thus, LC3 II may represent the number of autophagosomes and is a good marker for their formation (27). The process of autophagy includes induction, nucleation, extension and formation of the membrane, formation of autophagosomes and fusion with lysosomes $(28,29)$. Autophagy-related protein Beclin-1, encoded by the BECN1 gene, is a major protein involved in the nucleation of autophagosomes, and is the key target for regulation of autophagy (30). Therefore, the authors investigated the levels of these two proteins in the model of RSLT. HO-1/BM-MSCs-treated group had the highest level of autophagy-related proteins LC3 II and Beclin-1, which suggested that significant amounts of autophagy occurred in the HO-1/BM-MSCs-treated group, and was consistent with ultrastructure results of the transplanted livers. Previous studies indicated that autophagy, as a cytoprotective mechanism, is involved in biological process such as growth, development and immune regulation (14,31). In addition, autophagy could relieve ischemia-reperfusion injury and induce immune tolerance in the organ transplantation $(16,17,32-37)$. On the one hand, ischemia-reperfusion injury involves many mechanisms, among which autophagy can remove damaged mitochondria to prevent the accumulation of abnormal mitochondria and toxic products in the cytoplasm (mitochondrial autophagy) (32). Swelling and structural damage of mitochondria in the liver of transgenic mice with autophagic defects have been reported (33).

Autophagy could also inhibit the release of proinflammatory cytokines, such as tumor necrosis factor (TNF)- $\alpha$, interleukin (IL)-2, IL-6 and IL-1, and the high mobility group proteins produced by activated Kupffer cells, neutrophils and platelets, to prevent the death of hepatocytes (15-17). TNF- $\alpha$ could lead to swelling of the endothelia and activation of ROS; IL-6 could damage hepatocytes, and induce them to produce C-reactive protein, $\alpha$-trypsin and fibrinogen (34). These observations were consistent with the authors' preliminary experimental results that $\mathrm{HO}-1 / \mathrm{BM}-\mathrm{MSC}$ could reduce the levels of IL-2 and TNF- $\alpha$ in the transplanted liver $(12,13)$.

On the other hand, autophagy may be involved in the induction of immune tolerance, among which thymic epithelial cells, professional and non-professional antigen presenting cells from the thymus and its periphery, could present autologous antigens to MHC II molecules directly via autophagy, subsequently promoting the central tolerance and peripheral immune tolerance of $\mathrm{CD} 4^{+} \mathrm{T}$ cells (35). Autophagy also inhibits the maturation of DCs, induces the expression of
$\mathrm{CD} 4{ }^{+} \mathrm{CD} 25^{+} \mathrm{Foxp}^{+} \mathrm{T}$ cells and $\mathrm{CD} 8^{+} \mathrm{CD} 25^{+} \mathrm{Foxp}^{+} \mathrm{T}$ cells, and increases the secretion of IL-10 to induce allogeneic T cell anergy $(36,37)$, which were consistent with the preliminary results suggesting that $\mathrm{HO}-1 / \mathrm{BM}-\mathrm{MSC}$ could increase IL-10 and regulatory $\mathrm{T}$ cells in the transplanted liver $(12,13)$. All of these effects of autophagy in the field of organ transplantation were similar to those induced by BM-MSCs.

BM-MSCs could also reduce ischemia-reperfusion injury by secretion of cytokines and showed immunomodulatory effects $(8,9,38,39)$. A previous study demonstrated a link between the effects of BM-MSCs and autophagy (40). In addition, induction of HO-1 is an adaptive response to recover cell homeostasis (such as autophagy) (26). HO-1 increases the signals of autophagy, and inhibition of HO-1 activity or HO-1 knockout led to reduced autophagy in hepatocytes, with increased cell death; inhibition of autophagy also weakened the anti-inflammatory effects of HO-1 (41-43). Therefore, autophagy and expression of autophagy-related proteins are associated closely with BM-MSCs and HO-1.

In the present study, the authors further found that autophagy was enhanced and apoptosis was weakened in the HO-1/BM-MSCs-treated group, which suggested that autophagy is involved in the protective effects of HO-1/BM-MSCs on transplanted livers following RSLT. In addition, the amount of LC3 II protein was higher on POD 1 than on POD 3, while it was lower on POD 14 than POD 10. The authors further analyzed the mechansim. Previous studies have found that starvation is a potent inducer of antophagy (44). In response to starvation, autophagy in the liver of rodents increased, and the rate of protein degradation was also increased to maintain cell function (14). As a result, the authors believe that this phenomenon probably correlates with starvation of the transplanted liver, since the recipient eats less food on POD 1. As the degree of rejection increased over time, the autophagy of remnant hepatocytes was not sufficient to counter the severe rejection on POD 14, so the amount of LC3 II protein was lower on POD 14 than POD 10.

Autophagy is a complex process that is tightly regulated by $>30$ autophagy-related proteins, and by signaling pathways such as the mammalian target of rapamycin (mTOR), adenosine monophosphate-activated protein kinase and hypoxia-inducible factor pathways (45). Among them, mTOR is an important receptor of intracellular energy and nutritional status. mTOR is an evolutionarily conserved serine/threonine protein kinase, and is a negative regulator of autophagy proteins. It comprises two different complexes: mTORC1 and mTORC2 (46). mTORC1 is the focus of many upstream stimuli and signals, including MEK/ERK and PI3K/AKT, which regulate autophagy and other cellular activities (46). Among them, ERK is a member of the mitogen-activated protein kinase family and responds to both intracellular and extracellular stimuli. p-ERK regulates cytoskeletal proteins, kinases and transcription factors, leading to altered gene expression, cell proliferation and differentiation (47-49). Therefore, the ERK/mTOR signaling pathway was chosen to explore its possible involvement in the mechanism of autophagy associated with the protective effects of HO-1/BM-MSCs on transplanted livers after RSLT. The present study demonstrated increased p-ERK and decreased p-mTOR in the BM-MSCs-treated and HO-1/BM-MSCs-treated groups. 
The results in the HO-1/BM-MSCs-treated group were more dramatic. Activation of MEK/ERK and inhibition of mTORC1 could enhance the expression of autophagy-related proteins, leading to increased autophagic activity, which could protect cells $(50,51)$. The results of the present study demonstrated that ERK is a negative regulator of the upstream mTORC1, and autophagy may have protective effects on cells via this signaling pathway. Therefore, it is hypothesized that autophagy is involved in the protective effects of HO-1/BM-MSCs on the transplanted liver after RSLT through the ERK/mTOR signaling pathway.

To the best of the authors' knowledge, the present study is the first to demonstrate that autophagy is involved in the protective effects of HO-1/BM-MSCs on the transplanted liver after RSLT. Regulation of autophagy is primarily mediated through the ERK/mTOR signaling pathway. In a future study, the authors intend to carry out related experiments (such as the use of inhibitors, inducers and pathway blockers of autophagy, autophagy-related gene knockout animal models). Future studies will also focus on additional in vitro experiments to provide a theoretical basis for the reduction of transplanted liver damage after RSLT and its wider clinical application.

\section{Acknowledgements}

The authors would like to thank the Key Laboratory of the Emergency and Care Medicine of Ministry of Health and Tianjin Key Laboratory of Organ Transplantation (Tianjin, China) for allowing this work to progress in their laboratories. The present study was supported by the National Natural Science Foundation of China (grant nos. 81670574, 81441022 and 81270528); the Natural Science Foundation of Tianjin, China (grant nos. 08JCYBJC08400, 11JCZDJC27800 and 12JCZDJC25200); and the Technology Foundation of the Health Bureau in Tianjin, China (grant no. 2011KY11).

\section{References}

1. Müller SA, Mehrabi A, Schmied BM, Welsch T, Fonouni H, Engelmann G, Schemmer P, Weitz J and Schmidt J: Partial liver transplantation-living donor liver transplantation and split liver transplantation. Nephrol Dial Transplant 22 (Suppl 8): viii13-viii22, 2007.

2. Dahm F, Georgiev P and Clavien PA: Small-for-size syndrome after partial liver transplantation: Definition, mechanisms of disease and clinical implications. Am J Transplant 5: 2605-2610, 2005.

3. Taki-Eldin A, Zhou L, Xie HY and Zheng SS: Liver regeneration after liver transplantation. Eur Surg Res 48: 139-153, 2012.

4. Goss JA, Yersiz H, Shackleton CR, Seu P, Smith CV, Markowitz JS, Farmer DG, Ghobrial RM, Markmann JF, Arnaout WS, et al: In situ splitting of the cadaveric liver for transplantation. Transplantation 64: 871-877, 1997.

5. Davani S, Marandin A, Mersin N, Royer B, Kantelip B, Hervé P, Etievent JP and Kantelip JP: Mesenchymal progenitor cells differentiate into an endothelial phenotype, enhance vascular density, and improve heart function in a rat cellular cardiomyoplasty model. Circulation 108 (Suppl 1): II253-II258, 2003.

6. Popp FC, Piso P, Schlitt HJ and Dahlke MH: Therapeutic potential of bone marrow stem cells for liver diseases. Curr Stem Cell Res Ther 1: 411-418, 2006.

7. Zhou QI, Yang C and Yang P: The promotional effect of mesenchymal stem cell homing on bone tissue regeneration. Curr Stem Cell Res Ther, 2015.

8. Ren G, Zhang L, Zhao X, Xu G, Zhang Y, Roberts AI, Zhao RC and Shi Y: Mesenchymal stem cell-mediated immunosuppression occurs via concerted action of chemokines and nitric oxide. Cell Stem Cell 2: 141-150, 2008
9. Spaggiari GM, Abdelrazik H, Becchetti F and Moretta L: MSCs inhibit monocyte-derived DC maturation and function by selectively interfering with the generation of immature DCs: Central role of MSC-derived prostaglandin E2. Blood 113: 6576-6583, 2009.

10. Huang HF, Zeng Z, Wang KH, Zhang HY, Wang S, Zhou WX, Wang $\mathrm{ZB}, \mathrm{Xu}$ WG and Duan J: Heme oxygenase-1 protects rat liver against warm ischemia/reperfusion injury via TLR2/ TLR4-triggered signaling pathways. World J Gastroenterol 21: 2937-2948, 2015 .

11. Zeng B, Lin G, Ren X, Zhang Y and Chen H: Over-expression of HO-1 on mesenchymal stem cells promotes angiogenesis and improves myocardial function in infarcted myocardium. $\mathrm{J}$ Biomed Sci 17: 80, 2010.

12. Shen ZY, Wu B, Liu T, Yang Y, Yin ML, Zheng WP, Zhang BY and Song HL: Immunomodulatory effects of bone marrow mesenchymal stem cells overexpressing heme oxygenase-1: Protective effects on acute rejection following reduced-size liver transplantation in a rat model. Cell Immunol 313: 10-24, 2017.

13. Wu B, Song HL, Yang Y, Yin ML, Zhang BY, Cao Y, Dong C and Shen ZY: Improvement of liver transplantation outcome by heme oxygenase-1-transduced bone marrow mesenchymal stem cells in rats. Stem Cells Int 2016: 9235073, 2016.

14. Czaja MJ, Ding WX, Donohue TM Jr, Friedman SL, Kim JS, Komatsu M, Lemasters JJ, Lemoine A, Lin JD, Ou JH, et al: Functions of autophagy in normal and diseased liver. Autophagy 9: 1131-1158, 2013

15. Li L, Tan J, Miao Y, Lei P and Zhang Q: ROS and autophagy: Interactions and molecular regulatory mechanisms. Cell Mol Neurobiol 35: 615-621, 2015 .

16. Liu J, Hao H, Huang H, Tong C, Ti D, Dong L, Chen D, Zhao Y, Liu H, Han W, et al: Hypoxia regulates the therapeutic potential of mesenchymal stem cells through enhanced autophagy. Int J Low Extrem Wounds 14: 63-72, 2015.

17. Bhogal RH, Weston CJ, Curbishley SM, Adams DH and Afford SC: Autophagy: A cyto-protective mechanism which prevents primary human hepatocyte apoptosis during oxidative stress. Autophagy 8: 545-558, 2012.

18. Klein L, Münz C and Lünemann JD: Autophagy-mediated antigen processing in $\mathrm{CD}^{+} \mathrm{T}$ cell tolerance and immunity. FEBS Lett 584: 1405-1410, 2010.

19. Zhao Q, Ren H, Zhu D and Han Z: Stem/progenitor cells in liver injury repair and regeneration. Biol Cell 101: 557-571, 2009.

20. [No authors listed]: Banff schema for grading liver allograft rejection: An international consensus document. Hepatology 25: 658-663, 1997.

21. Livak KJ and Schmittgen TD: Analysis of relative gene expression data using real-time quantitative PCR and the 2(-Delta Delta C(T)) Method. Methods 25: 402-408, 2001.

22. van Poll D, Parekkadan B, Cho CH, Berthiaume F, Nahmias Y, Tilles AW and Yarmush ML: Mesenchymal stem cell-derived molecules directly modulate hepatocellular death and regeneration in vitro and in vivo. Hepatology 47: 1634-1643, 2008.

23. Rabani V, Shahsavani M, Gharavi M, Piryaei A, Azhdari Z and Baharvand $\mathrm{H}$ : Mesenchymal stem cell infusion therapy in a carbon tetrachloride-induced liver fibrosis model affects matrix metalloproteinase expression. Cell Biol Int 34: 601-605, 2010.

24. Fouraschen SM, Wolf JH, van der Laan LJ, de Ruiter PE, Hancock WW, van Kooten JP, Verstegen MM, Olthoff KM and de Jonge J: Mesenchymal stromal cell-derived factors promote tissue repair in a small-for-size ischemic liver model but do not protect against early effects of ischemia and reperfusion injury. $\mathrm{J}$ Immunol Res 2015: 202975, 2015.

25. Otterbein LE, Soares MP, Yamashita K and Bach FH: Heme oxygenase-1: Unleashing the protective properties of heme. Trends Immunol 24: 449-455, 2003.

26. Waltz P, Carchman EH, Young AC, Rao J, Rosengart MR, Kaczorowski D and Zuckerbraun BS: Lipopolysaccaride induces autophagic signaling in macrophages via a TLR4, heme oxygenase-1 dependent pathway. Autophagy 7: 315-320, 2011.

27. Tanida I, Minematsu-Ikeguchi N, Ueno T and Kominami E: Lysosomal turnover, but not a cellular level, of endogenous LC3 is a marker for autophagy. Autophagy 1: 84-91, 2005.

28. Loos B, Engelbrecht AM, Lockshin RA, Klionsky DJ and Zakeri Z: The variability of autophagy and cell death susceptibility: Unanswered questions. Autophagy 9: 1270-1285, 2013.

29. Komatsu M: Liver autophagy: Physiology and pathology. J Biochem 152: 5-15, 2012. 
30. Klionsky DJ, Abdelmohsen K, Abe A, Abedin MJ, Abeliovich $\mathrm{H}$, Acevedo Arozena A, Adachi H, Adams CM, Adams PD, Adeli K, et al: Guidelines for the use and interpretation of assays for monitoring autophagy (3rd edition). Autophagy 12: 1-222, 2016.

31. Liu Q, Sun Y, Lv Y, Le Z, Xin Y, Zhang P and Liu Y: TERT alleviates irradiation-induced late rectal injury by reducing hypoxia-induced ROS levels through the activation of NF- $\mathrm{BB}$ and autophagy. Int J Mol Med 38: 785-793, 2016.

32. Lee S and Kim JS: Mitophagy: Therapeutic potentials for liver disease and beyond. Toxicol Res 30: 243-250, 2014.

33. Takamura A, Komatsu M, Hara T, Sakamoto A, Kishi C, Waguri S, Eishi Y, Hino O, Tanaka K and Mizushima N: Autophagy-deficient mice develop multiple liver tumors. Genes Dev 25: 795-800, 2011.

34. Shen M, Lu J, Dai W, Wang F, Xu L, Chen K, He L, Cheng P, Zhang Y, Wang C, et al: Ethyl pyruvate ameliorates hepatic ischemia-reperfusion injury by inhibiting intrinsic pathway of apoptosis and autophagy. Mediators Inflamm 2013: 461536 2013.

35. Lünemann JD and Münz C: Autophagy in $\mathrm{CD} 4^{+} \mathrm{T}$-cell immunity and tolerance. Cell Death Differ 16: 79-86, 2009.

36. Li X, Li JJ, Yang JY, Wang DS, Zhao W, Song WJ, Li WM, Wang JF, Han W, Zhang ZC, et al: Tolerance induction by exosomes from immature dendritic cells and rapamycin in a mouse cardiac allograft model. PLoS One 7: e44045, 2012.

37. Li XC, Strom TB, Turka LA and Wells AD: T cell death and transplantation tolerance. Immunity 14: 407-416, 2001.

38. Isbambetov A, Baimakhanov Z, Soyama A, Hidaka M, Sakai Y Takatsuki M, Kuroki T and Eguchi S: Equal distribution of mesenchymal stem cells after hepatic ischemia-reperfusion injury. J Surg Res 203: 360-367, 2016.

39. Jin G, Qiu G, Wu D, Hu Y, Qiao P, Fan C and Gao F: Allogeneic bone marrow-derived mesenchymal stem cells attenuate hepatic ischemia-reperfusion injury by suppressing oxidative stress and inhibiting apoptosis in rats. Int J Mol Med 31: 1395-1401, 2013.

40. Nuschke A, Rodrigues M, Stolz DB, Chu CT, Griffith L and Wells A: Human mesenchymal stem cells/multipotent stromal cells consume accumulated autophagosomes early in differentiation. Stem Cell Res Ther 5: 140, 2014
41. Yun N, Cho HI and Lee SM: Impaired autophagy contributes to hepatocellular damage during ischemia/reperfusion: Heme oxygenase-1 as a possible regulator. Free Radic Biol Med 68: 168-177, 2014.

42. Carchman EH, Rao J, Loughran PA, Rosengart MR and Zuckerbraun BS: Heme oxygenase-1-mediated autophagy protects against hepatocyte cell death and hepatic injury from infection/sepsis in mice. Hepatology 53: 2053-2062, 2011

43. Wang Y, Xiong X, Guo H, Wu M, Li X, Hu Y, Xie G, Shen J and Tian Q: ZnPP reduces autophagy and induces apoptosis, thus aggravating liver ischemia/reperfusion injury in vitro. Int J Mol Med 34: 1555-1564, 2014.

44. Deretic V, Saitoh T and Akira S: Autophagy in infection, inflammation and immunity. Nat Rev Immunol 13: 722-737, 2013.

45. White E, Mehnert JM and Chan CS: Autophagy, metabolism, and cancer. Clin Cancer Res 21: 5037-5046, 2015.

46. Yang Z and Klionsky DJ: Mammalian autophagy: Core molecular machinery and signaling regulation. Curr Opin Cell Biol 22: 124-131, 2010.

47. Ma K, Huang MY, Guo YX and Hu GQ: Matrine-induced autophagy counteracts cell apoptosis via the ERK signaling pathway in osteosarcoma cells. Oncol Lett 12: 1854-1860, 2016.

48. $\mathrm{Lu} \mathrm{Z}$ and Xu S: ERK1/2 MAP kinases in cell survival and apoptosis. IUBMB Life 58: 621-631, 2006.

49. Wen J, Zhao Y and Guo L: Orexin A induces autophagy in HCT-116 human colon cancer cells through the ERK signaling pathway. Int J Mol Med 37: 126-132, 2016.

50. Wang PR, Wang JS, Zhang C, Song XF, Tian N and Kong LY: Huang-Lian-Jie-Du-Decotion induced protective autophagy against the injury of cerebral ischemia/reperfusion via MAPK-mTOR signaling pathway. J Ethnopharmacol 149: 270-280, 2013

51. Yang J, Chen Q, Tian S, Song S, Liu F, Wang Q and Fu Z: The role of 1,25-dyhydroxyvitamin D3 in mouse liver ischemia reperfusion injury: Regulation of autophagy through activation of MEK/ ERK signaling and PTEN/PI3K/Akt/mTORC1 signaling. Am J Transl Res 7: 2630-2645, 2015. 